\title{
Concentration of Vacancies at Metal-Oxide Surfaces: Case Study of $\operatorname{MgO}(100)$
}

\author{
Norina A. Richter, ${ }^{1}$ Sabrina Sicolo, ${ }^{2}$ Sergey V. Levchenko, ${ }^{1}$ Joachim Sauer, ${ }^{2}$ and Matthias Scheffler ${ }^{1}$ \\ ${ }^{1}$ Fritz-Haber-Institut der Max-Planck-Gesellschaft, Faradayweg 4-6, 14195 Berlin, Germany \\ ${ }^{2}$ Institut für Chemie, Humboldt Universität zu Berlin, Unter den Linden 6, 10099 Berlin, Germany
}

(Received 2 May 2013; published 26 July 2013)

\begin{abstract}
We investigate the effects of doping on the formation energy and concentration of oxygen vacancies at a metal-oxide surface, using $\mathrm{MgO}(100)$ as an example. Our approach employs density-functional theory, where the performance of the exchange-correlation functional is carefully analyzed, and the functional is chosen according to a condition on density-functional theory ionization energies. The approach is further validated by coupled-cluster calculations, including single, double, and perturbative triple substitutions, for embedded clusters. We demonstrate that the concentration of oxygen vacancies at a doped oxide surface is largely determined by the formation of a macroscopically extended space-charge region.
\end{abstract}

DOI: 10.1103/PhysRevLett.111.045502

PACS numbers: 61.72.Bb, 61.72.jd, 68.47.Gh, 68.55.Ln

Metal oxides are key materials for many technological applications. For example, $\mathrm{MgO}$ is used as a catalyst for methane oxidation, $\mathrm{TiO}_{2}$ plays an important role as a photocatalyst, and $\mathrm{RuO}_{2}$ catalyzes the oxidation of carbon monoxide. It is generally accepted that intrinsic point defects, in particular oxygen vacancies (also called $F$ or color centers), play a decisive role in catalysis at oxide surfaces [1-4], but significant controversy exists regarding their formation energy, concentration, and charge state. In this Letter, we study these issues for $\mathrm{MgO}$ bulk and the $\mathrm{MgO}(100)$ surface in contact with an $\mathrm{O}_{2}$ gas phase at realistic temperature and pressure. Furthermore, we consider that realistic metal oxides are typically doped, either intentionally or unintentionally. Although the experimental band gap of $\mathrm{MgO}$ is $7.8 \mathrm{eV}$ [5], realistic samples are typically neither clear transparent nor insulating. Defects such as intrinsic point defects, impurities, and defect complexes can give rise to electron or hole conductivity $[1,6,7]$. In the following, we neglect defect complexes (e.g., dopant plus vacancy). Thus, for our study the role of dopants is to create a Fermi level, i.e., a reservoir for electrons and holes. This is termed "the global effect of doping." We focus our discussion on $p$-type $\mathrm{MgO}$, since it exhibits intriguing catalytic properties $[1,8,9]$. Still, our theoretical model covers also $n$-type material, where the concentration of $F$ centers is predicted to be negligible. Our main finding is that for $p$-type samples, surface $\mathrm{O}$ vacancies get doubly positively charged, which lowers their formation energy and results in a significant defect concentration. In fact, the free energy of formation in thermodynamic equilibrium is negative under typical catalytic temperatures and pressures. We show that the limiting factor to formation of

Published by the American Physical Society under the terms of the Creative Commons Attribution 3.0 License. Further distribution of this work must maintain attribution to the author(s) and the published article's title, journal citation, and DOI. surface oxygen vacancies is the formation of a spacecharge region (Fig. 1). Although well known for doped semiconductor surfaces, the effect of space charge, and the resulting band bending, on the concentration of surface defects has not been discussed so far.

The values of calculated defect energy levels and total energies are sensitive to the employed treatment of exchange and correlation (xc) of the many-electron system. Therefore, special attention is given below to this aspect. Our approach is inspired by Slater's $X \alpha$ concept $[10,11]$ : We determine the best xc functional of the Heyd-ScuseriaErnzerhof (HSE) family by the condition that the ionization potentials obtained as total-energy differences with the optimized (opt-) HSE functional agree with the results of a $G_{0} W_{0}$ calculation using the same opt-HSE as input.

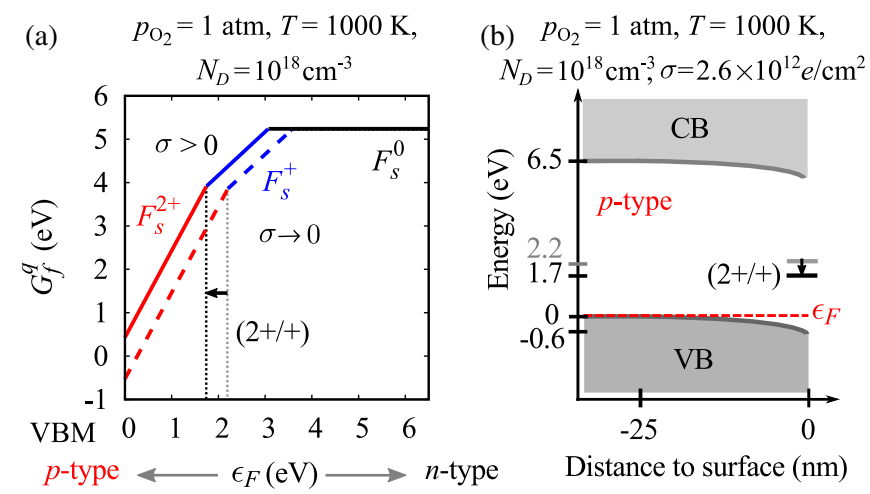

FIG. 1 (color online). (a) Calculated Gibbs free energies of formation $G_{f}^{q}$ (see text) of $F$ centers at $\mathrm{MgO}(100)$ for $T=$ $1000 \mathrm{~K}$ and normal pressure of oxygen, as a function of Fermi energy $\epsilon_{F}$, where the energy zero is at the valence-band maximum (VBM). Realistic dopant concentration $N_{D}=10^{18} \mathrm{~cm}^{-3}$ and surface charge density $\sigma=2.6 \times 10^{12} \mathrm{e} / \mathrm{cm}^{2}$ (solid lines) and the dilute limit $\sigma \rightarrow 0$ (dashed lines) are shown. (b) In $p$-type $\mathrm{MgO}\left(\epsilon_{F}=\mathrm{VBM}\right)$ under realistic conditions, band bending, due to formation of a space-charge region, limits the formation of surface $F_{s}^{2+}$ centers. 
In terms of the HSE [12] exchange and range-separation parameters $(\alpha, \omega)$, the Perdew-Burke-Ernzerhof (PBE) [13] generalized gradient approximation corresponds to (0, arbitrary), the HSE06 [14] hybrid functional to $\left(0.25,0.11 \mathrm{bohr}^{-1}\right)$, and the PBE0 [15] hybrid functional to $(0.25,0)$. Formation energies of neutral defects are validated using coupled-cluster theory with single and double substitutions, treating triple substitutions by perturbation theory, $\operatorname{CCSD}(\mathrm{T})[16,17]$, for embedded $\mathrm{Mg}_{x} \mathrm{O}_{y}$ clusters.

We use the all-electron FHI-aims code [18] for periodic structures (bulk and surfaces) and some embedded cluster calculations. FHI-aims employs atom-centered numeric basis functions and various xc functionals as well as the $G W$ approach [19]. The basis set and numerical grids are of high quality as defined by the tight [18] settings. For periodic models full atomic relaxation is calculated using PBE at the PBE bulk lattice parameter (4.258 $\AA$ ). HSE calculations are performed at these geometries. Using HSE geometries for two smallest unit cells considered results in negligible changes in the calculated formation energies. Vibrational energy $\Delta F_{\text {vib }}^{q}(T)$ and van der Waals contributions to the formation energies were analyzed as well [20], but found to be insignificant for this study.

We also employ the TURBOMOLE program package [21] for various embedded clusters using different $\mathrm{xc}$ functionals and $\operatorname{CCSD}(\mathrm{T})$. Triple-zeta valence plus polarization basis sets $[5 s 3 p 2 d 1 f] /[5 s 4 p 3 d]$ are used [22]. For the $\operatorname{CCSD}(\mathrm{T})$ computations we correlate also electrons in the $\mathrm{Mg} 2 s$ and $2 p$ shells, using correlation-consistent polarized-core-valence $X$-tuple-zeta basis sets, cc-pCVXZ $(X=\mathrm{D}, \mathrm{T}, \mathrm{Q})$. On the $\mathrm{O}^{2-}$ ions we use the augmented correlation-consistent polarized-valence basis sets aug-cc$\mathrm{pVXZ}$ [23]. In both $\operatorname{CCSD}(\mathrm{T})$ and density-functional theory (DFT) calculations, the basis set superposition error is evaluated following the Boys-Bernardi counterpoise correction [24]. $\mathrm{MgO}$ clusters are embedded in a point charge array using the periodic electrostatic embedded cluster model [25] in TURBOMOLE and a converged finite set of point charges in FHI-aims. (See Supplemental Material at [26] for more details.) To minimize nonphysical polarization of peripheral oxygen anions by the embedding point charges, pseudopotentials were added to the first shell of embedding $\mathrm{Mg}^{2+}$ cations (all-electron Hay and Wadt effective core potentials [27] in TURBOMOLE, and Troullier-Martins-type norm-conserving nonlocal pseudopotentials $[28,29]$ in FHI-aims). The PBE lattice constant has been used for the embedded clusters. Except for the CCSD(T) and $G W$ calculations and respective DFT values, atomic relaxation is allowed for all but outermost atoms in the cluster calculations.

In realistic samples, defects may get charged due to electron transfer between the dopant-induced Fermi level and the defect states [30]. The neutral oxygen vacancy in $\mathrm{MgO}$ bulk and at the $\mathrm{MgO}(100)$ surface has an energy level deep in the band gap. This state has $s$-like symmetry at the defect site, and is fully occupied by two electrons. Thus, a singly and a doubly charged vacancy is possible. In FHIaims this situation can be modeled in two ways: either by adding a constant charge density to the density entering the Hartree term, or by slightly modifying the nuclear charges of the atoms in the unit cell. Either concept enables us to describe a charged defect while the supercell is kept neutral. The "constant-density approach" is the standard treatment in other periodic codes. For surfaces, where much of the supercell corresponds to vacuum, this approach is obviously unphysical, although it can be partially remedied by $a$ posteriori correction schemes [31]. The other treatment corresponds to the virtual-crystal approximation (VCA) $[32,33]$ of a crystal with dopants. We change the nuclear number of all $\mathrm{Mg}$ atoms in the supercell by $\Delta Z_{\mathrm{Mg}}=$ $-q / N_{\mathrm{Mg}}$, where $q$ is the charge of the oxygen vacancy $\left(+1\right.$ or +2 ), and $N_{\mathrm{Mg}}$ is the number of $\mathrm{Mg}$ atoms in the supercell. This means that 1 or 2 electrons are transferred to the valence band maximum (VBM), which is the Fermi level in the virtual crystal. Once known for one particular Fermi level, the $\mathrm{O}$ vacancy formation energy can be trivially calculated for an arbitrary Fermi level [see Eq. (1)].

When removing atoms from the bulk or from the surface of a material, we need to consider also a reservoir to which the atoms are brought [34,35]. We assume a gas phase of $\mathrm{O}_{2}$ molecules, which is characterized by an oxygen chemical potential, $\mu_{\mathrm{O}}(T, p)$ [35]. For an isolated oxygen vacancy, the Gibbs free energy of formation is

$$
G_{f}^{q}=E_{\mathrm{vac}}^{q}-E_{\mathrm{host}}+\mu_{\mathrm{O}}+q \epsilon_{F}+\Delta F_{\mathrm{vib}}^{q}(T) .
$$

Here, $E_{\mathrm{vac}}^{q}$ and $E_{\text {host }}$ are DFT total energies of defected and undefected systems, respectively, $\Delta F_{\text {vib }}^{q}$ is the change in vibrational Helmholtz free energy of the crystal upon defect formation, $q$ is the defect charge, and $\epsilon_{F}$ is the Fermi energy. The oxygen chemical potential is

$$
\mu_{\mathrm{O}}=E_{\mathrm{O}}-\frac{1}{2} E_{\mathrm{O}_{2}}^{\text {bind }}+\Delta \mu_{\mathrm{O}},
$$

where $\Delta \mu_{\mathrm{O}}$ contains the vibrational and other $T$ - and $p$-dependent terms [35]. We use the experimental binding energy without zero point energies $E_{\mathrm{O}_{2}}^{\text {bind }}=5.22 \mathrm{eV}$ [36] to reduce artifacts originating from the generalized gradient approximations for the binding of the $\mathrm{O}_{2}$ molecule. The total energy of the free atom $E_{\mathrm{O}}$, is calculated with the corresponding electronic-structure approach (see the Supplemental Material [26]). $\Delta \mu_{\mathrm{O}}=0$ defines the oxygen-rich limit.

First, we address formation energies for isolated vacancies in the bulk $G_{f}^{\text {bulk, } q}$. We extrapolate our DFT formation energies to the dilute limit using Taylor expansion in terms of reciprocal supercell lattice constant. In agreement with related work [37], we find that the charge-transition levels $(2+/+)$ and $(+/ 0)$, as well as formation energies $G_{f}^{\mathrm{bulk}, q}$, are almost independent on the xc functional within the HSE family, when referenced to the vacuum level. 
However, given the more realistic situation of $p$-type material, where $\epsilon_{F}$ is at VBM, $G_{f}^{\text {bulk, } q}$ does depend strongly on the choice of HSE parameters $(\alpha, \omega)$ for $q \neq 0$. We find that for fixed $\omega$ the formation energies depend practically linearly on the exchange parameter $\alpha$, which can be traced back to a close-to-linear dependence of VBM with respect to vacuum on $\alpha$ (see also Ref. [38]).

Next, we identify the optimal xc functional to describe the formation energies of $F$ centers in $\mathrm{MgO}$. The ionization potential at a fixed defect geometry for a given functional HSE $(\alpha, \omega)$ is determined by the delta-self-consistent-field method $(\triangle \mathrm{SCF})$ :

$$
I_{\Delta \mathrm{SCF}}^{q \rightarrow q+1}=E_{\mathrm{vac}}^{q+1}+\epsilon_{F}-E_{\mathrm{vac}}^{q},
$$

where both $E_{\mathrm{vac}}^{q}$ and $E_{\mathrm{vac}}^{q+1}$ are extrapolated to the dilute limit. For $\epsilon_{F}=$ VBM, $I_{\Delta S C F}^{q \rightarrow q+1}$ depends on $(\alpha, \omega)$. In exact DFT the Kohn-Sham highest occupied orbital (HOMO) does not change with occupation and agrees with the ionization energy. A more practical request is that the HOMO, calculated by $G_{0} W_{0}$ on top of the HSE electronic ground state, should agree with the HSE ionization energy

$$
I_{G_{0} W_{0}}^{q \rightarrow q+1}=\epsilon_{F}-\epsilon_{\mathrm{HOMO}}^{G_{0} W_{0}} \stackrel{!}{=} I_{\Delta \mathrm{SCF}, \mathrm{opt}-\mathrm{HSE}}^{q \rightarrow q+1},
$$

identifying what we call the optimized HSE functional, opt-HSE that correctly describes the charge excitation of the defect.

We determine the opt-HSE functional for fixed $\omega=$ $0.11 \mathrm{bohr}^{-1}$. The ionization energies $I^{0 \rightarrow+}$ and $I^{+\rightarrow 2+}$ for $\epsilon_{F}$ at VBM are calculated at $F^{0}$ geometry for an embedded $\mathrm{Mg}_{6} \mathrm{O}_{9}$ cluster model using FHI-aims. The Fermi level $\epsilon_{F}$ is obtained as VBM $=E_{\text {host }}^{+1}-E_{\text {host }}$ using HSE, and from the HOMO of the host system in the corresponding $G_{0} W_{0} @$ HSE calculations. The ionization potentials show a near-linear dependence on the exchange parameter $\alpha$ (Fig. 2) for both $\triangle \mathrm{SCF}$ and $G W$ methods. The intersection of the linear fits are at $\alpha=0.27$ for $I^{0 \rightarrow+}$ and $\alpha=0.26$ for $I^{+\rightarrow 2+}$, very close to $\alpha$ in HSE06 $(\alpha=0.25)$. The same result is obtained, when the HSE ionization energies are determined from the highest occupied Kohn-Sham level at half occupation $[39,40]$. We therefore use HSE06 as our opt-HSE functional that correctly describes the charge excitation of the defect. The difference in formation energies with $\alpha=0.25$ instead of $\alpha=0.27$ is negligible for $F^{0}$, less than $0.1 \mathrm{eV}$ for $F^{+}$, and less than $0.2 \mathrm{eV}$ for $F^{2+}$.

We perform a validation for the $F^{0}$ formation energy using an unrelaxed embedded $\mathrm{Mg}_{6} \mathrm{O}_{9}$ cluster model and the $\operatorname{CCSD}(\mathrm{T})$ method. This results in a correction [41-44] $\triangle \mathrm{CCSD}(\mathrm{T})$ of the DFT formation energies of $-0.09 \mathrm{eV}$ for PBE, $0.07 \mathrm{eV}$ for PBE0, and $-0.28 \mathrm{eV}$ for the Becke, three parameter, Lee-Yang-Parr (B3LYP) functional. Adding these corrections to the DFT formation energies $\left(\Delta \mu_{\mathrm{O}}=0\right)$ for a converged cluster size $\mathrm{Mg}_{14} \mathrm{O}_{19}$ yields $\mathrm{DFT}+\Delta \operatorname{CCSD}(\mathrm{T})$ results of $6.85,6.88$, and $6.89 \mathrm{eV}$,

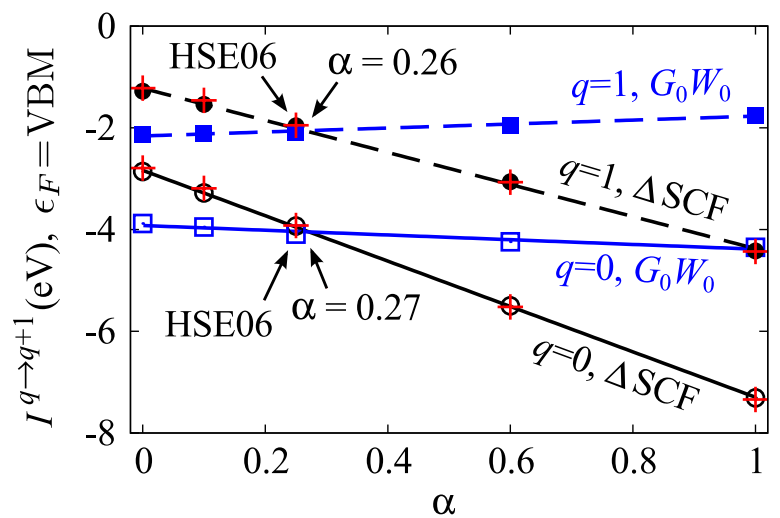

FIG. 2 (color online). Ionization potentials $I^{q \rightarrow q+1}$ at $F^{0}$ geometry calculated for an $\mathrm{Mg}_{6} \mathrm{O}_{9}$ embedded cluster by $\Delta \mathrm{SCF}$ with HSE xc functionals (dots and circles) and from the HOMO of a $G_{0} W_{0} @ \mathrm{HSE}$ calculation (filled and open squares). The screening parameter is $\omega=0.11 \mathrm{bohr}^{-1}$. Solid and dashed lines show linear fits to $I^{0 \rightarrow+}$ and $I^{+\rightarrow 2+}$ as a function of exchange parameter $\alpha$ (see the Supplemental Material [26] for additional information). Crosses show the Kohn-Sham eigenvalues at half occupation.

respectively. These numbers are in good agreement with the HSE06 $F^{0}$ formation energies 7.04 and $7.05 \mathrm{eV}$ obtained from the same converged embedded cluster and periodic calculations, respectively, using FHI-aims.

Thus, for MgO HSE06 is the opt-HSE functional in accordance with $G W$ as well as coupled-cluster results. Our results show that the experimental value for the bulk $F^{0}$ center formation enthalpy in $\mathrm{MgO}$ of $9.29 \mathrm{eV}$ with respect to the $\mathrm{O}_{2}$ molecule $[45,46]$ is a significant overestimate. A likely reason is that thermodynamic equilibrium has not been reached in this experiment.

We are now on solid grounds to provide an accurate estimate of $G_{f}^{q}$ for isolated oxygen vacancies at the surface using our periodic model and the HSE06 xc functional. $F_{s}^{0}$, $F_{s}^{+}$, and $F_{s}^{2+}$ formation energies in the dilute limit are $6.34 \mathrm{eV}, 2.76 \mathrm{eV}$, and $0.55 \mathrm{eV}$, respectively, for $\Delta \mu_{\mathrm{O}}=0$ and $\epsilon_{F}$ at VBM. For more realistic conditions, the formation energies are lower, as shown in Fig. 1(a). $G_{f}^{0}$ obtained with the CCSD(T) correction method is, as for the bulk, in good agreement with the HSE06 value. The corrections $\triangle \mathrm{CCSD}(\mathrm{T})$ to the DFT formation energies, calculated with an unrelaxed embedded $\mathrm{Mg}_{5} \mathrm{O}_{5}$ cluster, are $-0.26 \mathrm{eV}$ for PBE, $-0.01 \mathrm{eV}$ for PBE0, and $-0.28 \mathrm{eV}$ for B3LYP, yielding $\mathrm{DFT}+\Delta \operatorname{CCSD}(\mathrm{T})$ values of $6.23,6.25$, and $6.33 \mathrm{eV}$, respectively.

Formation energies of neutral $\mathrm{O}$ vacancies depend weakly on their concentration (up to approximately $3 \%$ for bulk and $12 \%$ for surface defects in $\mathrm{MgO}$ ). On the contrary, due to the slow decay of Coulomb interaction with distance, the formation energy $\mathrm{G}_{f}^{q}$ of charged defects will strongly depend on their concentration and the distribution of compensating charge. Thus, concentration of dopants $N_{D}$ and their distribution (doping profile) should have a strong effect on the defect formation energies. 
The equilibrium concentrations $\eta_{q}$ of oxygen vacancies in three different charge states $(q=0-2)$ are determined by the minimum of the total Gibbs free energy $G$ of the system with interacting defects,

$$
\frac{\partial G}{\partial \eta_{q}}=G_{f}^{q}\left(\eta_{0}, \eta_{1}, \eta_{2}\right)-T \frac{\partial s_{\mathrm{conf}}\left(\eta_{0}, \eta_{1}, \eta_{2}\right)}{\partial \eta_{q}}=0 .
$$

The configurational entropy $s_{\text {conf }}$ accounts for energetically degenerate arrangements of the defects (see the Supplemental Material [26]).

Surface defects are charged by accommodating charge carriers from the bulk. This results in depletion of the charge carriers and creation of a space-charge layer in the subsurface region. The resulting electrostatic potential causes band bending and prevents more charges from the bulk to reach the surface, increasing the energy cost per defect. As a result, there are two leading electrostatic contributions to the formation energy of charged defects: (i) attraction to the compensating charge, and (ii) band bending. The first contribution reduces the formation energy, while the second contribution increases it. The thickness of the space-charge layer $z^{\mathrm{SC}}$ depends on the doping profile, and may be limited by the thickness of the material. We consider the case of uniformly distributed dopants and unconstrained $z^{\mathrm{SC}}$, but the discussion can be straightforwardly generalized to the more complex situations. To stay focused on electrostatic effects, we also do not consider a possible $(T, p)$ dependence of the bulk charge carrier density $N_{e / h}^{\text {bulk. }}$ i.e., $N_{e / h}^{\text {bulk }} \equiv N_{D}$ is a constant external parameter.

The dependence of $G_{f}^{q}$ on the surface charge density $\sigma$ is calculated as follows. First, we calculate formation energies $G_{f}^{\mathrm{VCA}, q}\left(\sigma, z^{\mathrm{SC}}\right)$ at a fixed $z^{\mathrm{SC}}$, equal to the slab thickness $d$, using VCA (see the Supplemental Material [26]). The calculated formation energies include both electrostatic effects mentioned above. The actual $z^{\text {SC }}$ is determined by $N_{D}$ as follows:

$$
z^{\mathrm{SC}}=\frac{\sigma}{e N_{D}},
$$

where $e$ is the absolute value of the electron charge. The formation energy as a function of $\sigma$ is

$$
G_{f}^{\mathrm{VCA}, q}(\sigma)=G_{f}^{\mathrm{VCA}, q}(\sigma, d)-q E^{\mathrm{SC}}(\sigma, d)+q E^{\mathrm{SC}}\left(\sigma, z^{\mathrm{SC}}\right),
$$

where

$$
E^{\mathrm{SC}}\left(\sigma, z^{\mathrm{SC}}\right)=\frac{e \sigma}{6 \epsilon_{r} \epsilon_{0}} z^{\mathrm{SC}}
$$

is the classic expression for the energy of the space-charge (SC) region formation at a semiconductor surface. The temperature dependence of $z^{\mathrm{SC}}$ and $E^{\mathrm{SC}}\left(\sigma, z^{\mathrm{SC}}\right)$ at fixed $\sigma$ is neglected. The meaning of the last two terms in Eq. (7) is to replace the band bending contribution to the formation
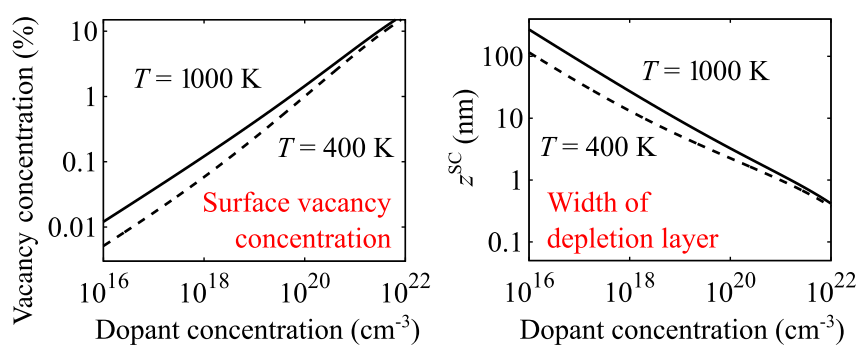

FIG. 3 (color online). Left: $F_{s}^{2+}$ concentration as a function of dopant concentration $N_{D}$ for two different temperatures and oxygen partial pressure of $1 \mathrm{~atm}$. Right: Dependence of the space-charge depth $z^{\mathrm{SC}}$ on $N_{D}$.

energy calculated for $z^{\mathrm{SC}}=d$ with the one obtained for the actual $z^{\mathrm{SC}}$ from Eq. (6). The remaining dependence on $\sigma$ after subtracting $q E^{\mathrm{SC}}(\sigma, d)$ from $G_{f}^{\mathrm{VCA}, q}(\sigma, d)$ is due to the electrostatic attraction between defect and compensating charge. The formation energy of a vacancy in charge state $q$ in the presence of other vacancies, entering Eq. (5), is then obtained as

$$
\mathrm{G}_{f}^{q}\left(\eta_{0}, \eta_{1}, \eta_{2}\right)=\frac{\partial}{\partial \eta_{q}} \sum_{r=0}^{2} \eta_{r} \mathrm{G}_{f}^{\mathrm{VCA}, r}(\sigma)
$$

with $\sigma=e \eta_{1}+2 e \eta_{2}$.

We can now calculate the equilibrium concentration of $\mathrm{O}$ vacancies at a $p$-doped $\mathrm{MgO}(100)$ surface, using Eq. (5). The concentrations of $F_{s}^{0}$ and $F_{s}^{+}$are found to be negligible at realistic $T, p_{\mathrm{O}_{2}}$, and $N_{D}$. The $F_{s}^{2+}$ concentration $\eta_{2}$ and corresponding $z^{\mathrm{SC}}$ as functions of $N_{D}$ are shown in Fig. 3 for different temperatures and $p_{\mathrm{O}_{2}}=1 \mathrm{~atm}$. Although the $F_{s}^{2+}$ Gibbs free energy of formation at $\sigma \rightarrow 0$ is small or even negative at elevated temperatures, the equilibrium defect concentration does not exceed $\sim 0.5 \%$ at $N_{D} \leq$ $10^{18} \mathrm{~cm}^{-3}$. Thus, space-charge layer formation can be a mechanism by which wide-band-gap semiconductor surfaces remain stable at high temperatures. The band bending profile for $\epsilon_{F}=\mathrm{VBM}$ at $T=1000 \mathrm{~K}$ for $p_{\mathrm{O}_{2}}=1 \mathrm{~atm}$ and $N_{D}=10^{18} \mathrm{~cm}^{-3}$ is shown in Fig. 1(b). Under these conditions the bulk bands bend downwards by $0.6 \mathrm{eV}$, and the $(2+/+)$ charge transition level is lowered from 2.2 to $1.7 \mathrm{eV}$ above the Fermi level. At $T=1000 \mathrm{~K}$ and $p_{\mathrm{O}_{2}}=$ $1 \mathrm{~atm}$, the contribution of the electrostatic attraction term is small for small $N_{D}$, but becomes comparable to $G_{f}^{2}(\sigma \rightarrow 0)$ for $N_{D}>10^{18} \mathrm{~cm}^{-3}$.

We have presented a methodology for calculating charged defect formation energies and concentrations at surfaces, taking into account electrostatic effects due to charge transfer between surface and bulk. Doping has been simulated using the VCA, and an optimal DFT functional has been identified by validation with coupled-cluster and $G W$ methods. Our analysis shows that the concentration of $F_{s}^{2+}$ centers at the (100) terrace of $p$-type $\mathrm{MgO}$ can be as high as $1 \%$ at realistic conditions, while relative $F_{s}^{+}$and 
$F_{s}^{0}$ concentrations are negligible. We demonstrate that the formation of charged vacancies creates a localized, although macroscopically extended, space-charge region. This lowers charge transition levels with respect to Fermi level at the surface, raising the vacancy formation energy by up to $1 \mathrm{eV}$ and, therefore, limiting the defect concentration. We conclude that doping can be used to tune the oxygen vacancy concentration at the surface of metal oxides. Experimental information on doping concentrations and profiles may provide new insights into catalytic activity of oxide surfaces.

This collaboration was financially supported by the cluster of excellence UniCat. N. A. R. acknowledges financial support from the International Max Planck Research School "Complex Surfaces in Materials Science." S. V. L. acknowledges financial support from the Alexander von Humboldt Foundation.

[1] I. Balint and K.-i. Aika, Appl. Surf. Sci. 173, 296 (2001).

[2] E. Wahlström, E. K. Vestergaard, R. Schaub, A. Ronnau, M. Vestergaard, E. Laegsgaard, I. Stensgaard, and F. Besenbacher, Science 303, 511 (2004).

[3] Z. Yan, S. Chinta, A. A. Mohamed, J.P. Fackler, and D. W. Goodman, J. Am. Chem. Soc. 127, 1604 (2005).

[4] D. O. Scanlon, A. Walsh, B. J. Morgan, and G. W. Watson, e-J. Surf. Sci. Nanotechnol. 7, 395 (2009).

[5] R. C. Whited and W. C. Walker, Phys. Rev. Lett. 22, 1428 (1969).

[6] S. P. Mitoff, J. Chem. Phys. 31, 1261 (1959).

[7] N. I. Hadi, J. Earth Sci. Clim. Change 03, 128 (2012).

[8] J.-L. Dubois and C. J. Cameron, Appl. Catal. 67, 49 (1990).

[9] S. Arndt, G. Laugel, S. Levchenko, R. Horn, M. Baerns, M. Scheffler, R. Schlögl, and R. Schomäcker, Catal. Rev. 53, 424 (2011).

[10] J. C. Slater and J.H. Wood, Int. J. Quantum Chem. 5, 3 (1970).

[11] J. C. Slater, in Advances in Quantum Chemistry, edited by Per-Olov Löwdin (Academic, New York, 1972), Vol. 6, pp. 1-92.

[12] J. Heyd, G.E. Scuseria, and M. Ernzerhof, J. Chem. Phys. 118, 8207 (2003).

[13] J. P. Perdew, K. Burke, and M. Ernzerhof, Phys. Rev. Lett. 77, 3865 (1996).

[14] A. V. Krukau, O. A. Vydrov, A. F. Izmaylov, and G. E. Scuseria, J. Chem. Phys. 125, 224106 (2006).

[15] J. P. Perdew, M. Ernzerhof, and K. Burke, J. Chem. Phys. 105, 9982 (1996).

[16] G. D. Purvis and R. J. Bartlett, J. Chem. Phys. 76, 1910 (1982).

[17] K. Raghavachari, G. W. Trucks, J. A. Pople, and M. HeadGordon, Chem. Phys. Lett. 157, 479 (1989).

[18] V. Blum, R. Gehrke, F. Hanke, P. Havu, V. Havu, X. Ren, K. Reuter, and M. Scheffler, Comput. Phys. Commun. 180, 2175 (2009); Fritz-Haber-Institut der Max-PlanckGesellschaft, Theory Department, "FHI-aims: FullPotential, All-Electron Electronic Structure Theory with
Numeric Atom-Centered Basis Functions," https:// aimsclub.fhi-berlin.mpg.de.

[19] X. Ren, P. Rinke, V. Blum, J. Wieferink, A. Tkatchenko, A. Sanfilippo, K. Reuter, and M. Scheffler, New J. Phys. 14, 053020 (2012).

[20] N. A. Richter, S. V. Levchenko, and M. Scheffler (to be published).

[21] TURBOMOLE V6.3 2011, a development of University of Karlsruhe and Forschungszentrum Karlsruhe $\mathrm{GmbH}$, 1989-2007, TURBOMOLE GmbH, since 2007.

[22] F. Weigend and R. Ahlrichs, Phys. Chem. Chem. Phys. 7, 3297 (2005).

[23] T. H. Dunning, Jr., J. Chem. Phys. 90, 1007 (1989).

[24] S.F. Boys and F.B. Bernardi, Mol. Phys. 19, 553 (1970).

[25] A. M. Burow, M. Sierka, J. Döbler, and J. Sauer, J. Chem. Phys. 130, 174710 (2009).

[26] See Supplemental Material at http://link.aps.org/ supplemental/10.1103/PhysRevLett.111.045502 for details on the embedded cluster and periodic calculations, and on the derivation for concentrations of surface defects.

[27] P. J. Hay and W. R. Wadt, J. Chem. Phys. 82, 270 (1985).

[28] L. Kleinman and D. M. Bylander, Phys. Rev. Lett. 48, 1425 (1982).

[29] M. Fuchs and M. Scheffler, Comput. Phys. Commun. 119, 67 (1999).

[30] C. Weinert and M. Scheffler, Mater. Sci. Forum 10-12, 25 (1986).

[31] H.-P. Komsa and A. Pasquarello, Phys. Rev. Lett. 110, 095505 (2013).

[32] L. Vegard, Z. Phys. 5, 17 (1921).

[33] M. Scheffler, Physica (Amsterdam) 146B+C, 176 (1987).

[34] M. Scheffler and J. Dabrowski, Philos. Mag. A 58, 107 (1988).

[35] K. Reuter, C. Stampfl, and M. Scheffler, in Handbook of Materials Modeling, edited by S. Yip (Springer, Dordrecht, Netherlands, 2005), Vol. 1, pp. 149-194.

[36] D. Feller and K. A. Peterson, J. Chem. Phys. 110, 8384 (1999).

[37] R. Ramprasad, H. Zhu, P. Rinke, and M. Scheffler, Phys. Rev. Lett. 108, 066404 (2012).

[38] D. West, Y. Y. Sun, and S. B. Zhang, Appl. Phys. Lett. 101, 082105 (2012).

[39] J.C. Slater, Quantum Theory of Molecules and Solids: The Self-Consistent Field for Molecules and Solids, International Series in Pure and Applied Physics Vol. 4 (McGraw-Hill, New York, 1974), 1st ed.

[40] J. F. Janak, Phys. Rev. B 18, 7165 (1978).

[41] C. Tuma and J. Sauer, Chem. Phys. Lett. 387, 388 (2004).

[42] C. Tuma and J. Sauer, Phys. Chem. Chem. Phys. 8, 3955 (2006).

[43] Q.-M. Hu, K. Reuter, and M. Scheffler, Phys. Rev. Lett. 98, 176103 (2007).

[44] Q.-M. Hu, K. Reuter, and M. Scheffler, Phys. Rev. Lett. 99, 169903(E) (2007).

[45] L. A. Kappers, R. L. Kroes, and E. B. Hensley, Phys. Rev. B 1, 4151 (1970).

[46] CRC Handbook of Chemistry and Physics, edited by R. C. Weast (CRC, Boca Raton, 1980), 61st ed. 